\title{
Propagation of Input Uncertainties in Numerical Simulations of Laser Powder Bed Fusion
}

\author{
SCOTT WELLS, ALEX PLOTKOWSKI, and MATTHEW JOHN M. KRANE
}

\begin{abstract}
Laser powder bed fusion has the potential of redefining state-of-the-art processing and production methods, but defect formation and inconsistent build quality have limited the implementation of this process. Numerical models are widely used to study this process and predict the formation of these defects. Presently, the uncertainties of model input parameters and thermophysical properties used by these numerical simulations have not been investigated. In the present study, the uncertainty in these input parameters and material properties are quantified for laser powder bed fusion, with and without a simulated powder bed, to determine their influence on the predictive accuracy of an experimentally validated numerical model. Accounting for all possible sources of uncertainty quickly becomes computationally expensive on account of the curse of dimensionality. Uncertainty in laser absorption, solid, and liquid specific heat of the metal were found to have the largest effect on model prediction reliability with or without the use of a powder bed. Results also illustrate that accounting for these three uncertain parameters still captures the majority of model prediction uncertainty. Furthermore, the methodology of this study may be used to understand the uncertainty in as-built microstructure through propagation to microstructure prediction models, or applied under processing conditions where high Péclet numbers are observed and the thermal convection and fluid flow within the molten pool are substantial.
\end{abstract}

https://doi.org/10.1007/s11663-021-02218-2

(C) The Minerals, Metals \& Materials Society and ASM International 2021

\section{INTRODUCTION}

LASER powder bed fusion (L-PBF) is a process through which a laser heat source, typically a neodymium-doped yttrium aluminum garnet (Nd:YAG) solid-state laser, ${ }^{[1]}$ scans a bed of metallic powder melting and consolidating the powder under the beam at a length scale on the order of 100 to 200 microns in diameter. To prevent combustion and reactions with the environment, the process is typically conducted in an inert atmosphere. By adding and locally melting multiple powder layers, complex geometries such as internal open-cell structures can be manufactured. Due to the small melt pool size, near net shaping of components is possible, reducing or eliminating the need for expensive post-production machining such as drilling or boring. Furthermore, the geometric flexibility of the process can reduce the number of parts needed in an assembly, thus

SCOTT WELLS and MATTHEW JOHN M. KRANE are with the School of Materials Engineering, Purdue Center of Metal Casting Research, Purdue University, West Lafayette, IN, 47907. Contact email: Wells130@purdue.edu ALEX PLOTKOWSKI is with the Materials Science and Technology Division, Oak Ridge National Laboratory, Oak Ridge, TN, 37831.

Manuscript submitted February 12, 2021, accepted April 4, 2021.

Article published online June 21, 2021. limiting the need for heavy fasteners and welding. Alloy systems most often used in the laser powder bed fusion process include: Ti-6Al-4V, SS 316, Inconel 718 and Inconel $625 .{ }^{[2]}$ These advantages and alloy systems make L-PBF attractive for use in the aerospace, automotive, and biomedical industries. ${ }^{[2]}$

One major factor limiting widespread production of components by laser powder bed fusion is inconsistencies in build quality. Parts exhibit variability in surface morphology $\mathrm{y}^{[3]}$ and defect formation (e.g., unintended keyholing, ${ }^{4,5]}$ porosity and cracking, ${ }^{[6-10]}$ lack of fusion ${ }^{[11]}$ and delamination $\left.{ }^{[12]}\right)$. These defects, and their variability make certifying components for critical applications produced by L-PBF difficult, as they degrade component mechanical properties. Consequently, minimization of these defects through process optimization and scan strategies, such as those described in Reference 13, is an important focus of laser welding and laser powder bed fusion research.

As detailed by DebRoy et al..${ }^{[2]}$ numerical simulations have been used extensively to provide insight into process parameter optimization by investigating predicted temperature histories and thermal gradients, melt pool flow, residual stress formation, and as-built grain morphology. These model outputs help establish processing windows in which the likelihood of defect formation is reduced. However, the physics of the 
process is very complex, featuring several modes of heat and mass transport, and a wide range of length and time scales which resolve behavior from the powder level up to the entire component. Full part simulations are computationally expensive and time consuming; therefore, simulations are often restricted to single line scans. The physical complexity and computational expense of the process models make their use in build design difficult, a difficulty exacerbated by the uncertainty in the input parameters and the choices made regarding the inclusion of physical phenomena and how to model them. The uncertainty in these input parameters (specifically, thermophysical properties, process parameters such as laser power, and model calibration variables such as laser absorption efficiency and penetration depth) propagate through the simulations into the melt pool dimensions and solidification time predictions which may then be further propagated to microstructural prediction models. Modeling of a component without some estimates of the uncertainties in the model outputs on which the design depends may lead to poor build quality and defect formation.

Presently, little consideration has been given to the uncertainty in model input parameters and what role it may have on the results of numerical simulations. One exception is work by Johnson et al. who used a trained surrogate model to quantify the uncertainty in printability maps for two alloy systems, based on the uncertainty in thermal conductivity and absorptivity of the alloys. ${ }^{[14]}$ Additionally, several efforts have been made to identify parameters that affect the build quality and component performance. Brandt ${ }^{[15]}$ identified more than 60 and Yagroitsev ${ }^{[16]}$ further increased this value to over 130 parameters for the laser powder bed fusion process. While most of these parameters are process specific, several serve as inputs to numerical simulations and heat transfer models. These model inputs will therefore be the primary focus of this uncertainty analysis.

This works' focus will was on a subset of parameters identified by Brand ${ }^{[15]}$ and Yagroitse ${ }^{[16]}$ that are inputs to the present numerical model. This reduction of order was effected by concentrating on conditions under which fluid flow within the molten pool has no appreciable influence on heat transfer and solidification. In realworld applications, this conduction-dominated regime is characterized by the non-dimensional Péclet number, which describes the ratio of convective to conductive heat transfer, being much less than 1, which is achieved by using low laser power and high scan speeds, yielding a low energy density within the molten pool. Under these conditions the surface tension gradients and the fluid motion they drive can be neglected, because previous experiments and computations have shown that flow has little influence on the shape of the melt pool or thermal behavior. ${ }^{[17]}$ This approximation is physically reasonable in our present cases and is numerically convenient as neglecting Marangoni flow reduces the number of uncertain input parameters. The scan speed and laser power used in the present study were assumed to fall within this regime based on the observations reported in Reference 17.

The primary model predictions of interest for this investigation were melt pool dimensions and solidification time where solidification time is defined as the time between the first solid forming and the last liquid solidifying. Melt pool dimensions are valuable when selecting heat source scan strategies for the laser powder bed fusion process due to the cyclical melting and heating experienced during a build, and to determine hatch spacing to avoid lack of fusion defects. Spatial microstructure variations, including grain size, phase fraction, and morphology, are often observed in as-built components. ${ }^{[18]}$ Understanding the dimensions of the molten pool enables more effective scan strategies to minimize these variations. Solidification time provides insight into the thermal gradients and cooling rates observed around the melt pool while also acting as an input to microstructural prediction models (e.g., Cellular Automata) to understand how the uncertainty of processing parameters propagate from the current model to the product of microstructure development simulations.

\section{METHODOLOGY}

In the present work, an experimentally validated finite volume method (FVM) model ${ }^{[17]}$ was applied to determine which parameters have the largest influence on the uncertainty in the predicted solidification time and melt pool dimensions during L-PBF of Inconel 625, given input uncertainty values estimated from the literature. The numerical model is coupled with TASMANIAN, ${ }^{[19-23]}$ a non-intrusive library for generating surrogate models for these outputs of interest. Monte Carlo (MC) sampling over the input uncertainties was applied to these surrogate models to produce probability distribution functions (PDF) for the simulation outputs of interest.

\section{A. Model Description}

\section{Numerical model}

For the present study, a finite volume method transport model based in OpenFOAM ${ }^{[17]}$ was used. This model is described in more detail in Reference 17 which also shows it has good agreement with melt pool shapes when the Péclet number is small (the conduction dominate regime). The current analysis was restricted to the conduction dominant regime, in which fluid velocity was assumed to be negligible in the prediction of heat transfer and solidification behavior. This assumption simplifies the governing equations, leaving only the conservation of energy:

$$
\frac{\partial\left(\rho c_{p} T\right)}{\partial t}=\nabla(k \nabla T)+S_{T}+\dot{Q}
$$


where $\rho$ is density, $c_{p}$ is specific heat, $k$ is thermal conductivity and $T$ is the temperature. The energy source term, $S_{\mathrm{T}}$, accounts for the latent heat release rate and is given by:

$$
S_{T}=-\left[\frac{\partial}{\partial t}\left(\rho f_{l} L_{f}\right)\right]
$$

where $f_{l}$ is the temperature-dependent liquid mass fraction calculated using a continuous sigmoidal function based on work by Rosler, ${ }^{[2]}$ and $L_{f}$ is the specific latent heat of melting. A Gaussian energy distribution is assumed for the volumetric heat source:

$$
\dot{Q}(x, y, z)=\frac{2 \eta P}{r^{2} d(\pi / 3)^{3 / 2}} \exp \left(\frac{-3\left(x^{2}+y^{2}\right)}{r^{2}}+\frac{-3 z^{2}}{d^{2}}\right)
$$

where $\eta$ is the laser absorption, $P$ is the simulated laser power, $r$ is the radius of the incident laser spot, and $d$ is the laser penetration depth into the material. The scan is along the $x$-axis and $z$ is the depth from the surface into the part. For this model, the laser absorption is not based on direct absorptivity measurements, rather, this parameter was calibrated to obtain the best agreement with experimental measurements for melt pool dimensions as discussed in Reference 17. The laser spot radius is constant at $85.7 \mu \mathrm{m}$. Although the actual penetration depth of lasers in metals and alloys is much smaller than the control volume size used, the penetration depth in the model was selected to spread the heat input over several control volumes, an assumption commonly used in laser welding research. As such, it is not a true representation of the physics, but rather the value of $d$ is assigned to enhance numerical stability. A more complete description of how these values were obtained can be found in Reference 17 along with a full description of the boundary conditions which are the same as used here.

Property data for density, specific heat, and thermal conductivity for bulk IN625 were obtained from JMatPro and used to solve the energy equation in the model. Neither the calculation method nor the associated uncertainties in these values is provided by JMatPro thus the uncertainty used in the present work were based on uncertainty values reported for the parameter specific measurement method. Table I summarizes the thermophysical property values obtained from JMatPro as functions of temperature.

When modeling the L-PBF process many researchers elect to not account for the topology or discrete nature of the powder bed. To quantitatively determine what effect the powder bed properties have on predictions of solidification time and melt pool dimensions, the present model was used for two separate analyses. Initially the properties used were those of fully dense Inconel 625 following the values given in Table I. The second analysis modeled the powder bed as a thin layer (30 $\mu \mathrm{m}$ ) of metal powder-gas mixture over a solid substrate. The effective density and specific heat of the simulated powder layer were modeled as volume weighted averages of the bulk and cover gas properties:

$$
\rho_{\text {eff }}(T, \phi)=\rho_{\text {gas }}(1-\phi)+\rho_{\text {metal }} \phi
$$

and

$$
c_{p, \text { eff }}(T, \phi)=c_{p, \text { gas }}(1-\phi)+c_{p, \text { metal }} \phi
$$

where the cover gas properties were that of argon to simulate an inert atmosphere. The powder packing density is $\phi$ and $1-\phi$ is the volume fraction filled with argon.

Thermal conductivity is poorly modeled as a weighted average because the paths and modes of heat transfer in heterogeneous porous media are different from those in a consolidated metal, where it is entirely by conduction. In powder beds, the contact area between particles is small and the contact and constriction resistances severely limit conductive heat flow. Conduction and radiation through the interpenetrating gas accounts for the majority of the thermal energy transport. Work by Gusarov and Kovalev ${ }^{[25]}$ concluded that the effective thermal conductivity of powder beds primarily depends

\begin{tabular}{|c|c|c|c|c|}
\hline Parameter & Units & Most Likely Values & Uncertainty $( \pm 3 \sigma)$ & Measurement Method \\
\hline$\rho_{S}$ & $\mathrm{~kg} / \mathrm{m}^{3}$ & $8604-0.6828 T$ & $1.0 \mathrm{pct}$ & dilatometry \\
\hline$\rho_{L}$ & $\mathrm{~kg} / \mathrm{m}^{3}$ & $8604-0.6828 T$ & $1.0 \mathrm{pct}$ & dilatometry \\
\hline$\rho_{\text {argon }}$ & $\mathrm{kg} / \mathrm{m}^{3}$ & $1.6954-3.6 \times 10^{-3} T+3 \times 10^{-6} T^{2}$ & $10 \mathrm{pct}$ & dilatometry \\
\hline $\mathrm{Cp}_{S}$ & $\mathrm{~J} / \mathrm{kg} \mathrm{K}$ & 754.77 & $4.0 \mathrm{pct}$ & calorimetry \\
\hline $\mathrm{Cp}_{L}$ & $\mathrm{~J} / \mathrm{kg} \mathrm{K}$ & 754.77 & $4.0 \mathrm{pct}$ & calorimetry \\
\hline $\mathrm{Cp}_{\text {argon }}$ & $\mathrm{J} / \mathrm{kg} \mathrm{K}$ & 520.0 & $4.0 \mathrm{pct}$ & calorimetry \\
\hline$k_{S}$ & $\mathrm{~W} / \mathrm{m} \mathrm{K}$ & $12.30+0.01472 T$ & $5.0 \mathrm{pct}$ & flash diffusivity \\
\hline$k_{L}$ & $\mathrm{~W} / \mathrm{m} \mathrm{K}$ & $8.961+0.01474 T$ & $5.0 \mathrm{pct}$ & flash diffusivity \\
\hline$k_{\text {argon }}$ & $\mathrm{W} / \mathrm{m} \mathrm{K}$ & $0.0249+7 \times 10^{-5} T$ & $5.0 \mathrm{pct}$ & flash diffusivity \\
\hline$L_{f}$ & $\mathrm{~J} / \mathrm{kg}$ & 217,500 & $4.0 \mathrm{pct}$ & calorimetry \\
\hline$P$ & W & 195 & $2.5 \mathrm{pct}$ & - \\
\hline$\eta$ & - & 0.31 & 15 pct & - \\
\hline$d$ & $\mu \mathrm{m}$ & 20 & 5.0 & - \\
\hline$\phi$ & - & 0.60 & 0.05 & - \\
\hline
\end{tabular}
on three morphological features: the volume fraction of the particles $(\phi)$, the average coordination number of

Table I. Most Likely Property Values and Uncertainties for Model Inputs

*(S), (L) correspond to solid and liquid IN625.

Uncertainties are assumed to follow a Gaussian distribution and temperature-dependences are expressed in ${ }^{\circ} \mathrm{C}$. 
Table II. Values Used to Calculate the Effective Conductivity of the Powder Bed, With Argon as the Gas Phase

\begin{tabular}{llll}
\hline$l$ & $68 \mathrm{~nm}$ & $\gamma_{\mathrm{Ar}}$ & $5 / 3$ \\
$D$ & $35 \mu \mathrm{m}$ & $\mathrm{Kn}$ & 0.0019 \\
$k_{A r}$ & $0.0249+\left(7 \times 10^{-5} / \mathrm{K}\right) T(\mathrm{~W} /$ & $\mathrm{L}$ & 59.4 \\
& $\mathrm{mK})$ & & \\
$N$ & 12 & $\frac{k_{\text {erf }}}{k_{\mathrm{Ar}}}$ & 12 \\
$\phi$ & 0.60 & & \\
\hline
\end{tabular}

Packed powder coordination number selected for the assumed FCC packing.

each particle $(N)$, and the neck-to-particle size ratio. They also defined a nondimensional characteristic length $(L)$, Eq. [8], as a function of $\phi, N$, the adiabatic index of the cover gas $(\gamma)$, and the dimensionless Knudsen number $(\mathrm{Kn})$ which is calculated from the mean-free path length $(l)$ and an assumed average particle diameter $(D)$. From these parameters, they derived an explicit formula to determine the effective thermal conductivity of a powder bed, showing good agreement with experimental data. This explicit formula, Eq. [9], was adapted in the present work with values given in Table II.

$$
\begin{gathered}
\gamma=c_{p} / c_{v} \\
K n=l / D \\
L=\left(\frac{\gamma+1}{9 \gamma+5}\right)\left(\frac{3}{4 \sqrt{\pi} K n}\right) \\
\frac{k_{\text {eff }}}{k_{\text {argon }}}=\frac{\phi N}{2}\left[\frac{1}{2} \ln (1+L)+\ln (1+\sqrt{L})+\frac{1}{1+\sqrt{L}}-1\right]
\end{gathered}
$$

For simulations of the laser powder bed fusion process, the density, specific heat, and thermal conductivity of each cell in the predefined powder layer follow Eqs. [4], [5], and [9], respectively. The properties were found from these equations until the liquid fraction of a given cell was calculated to be greater than 0.01 or 1 pct, which marked the onset of melting. Once this melting criterion was met, the cell was no longer considered powder, and the density, specific heat, and thermal conductivity values took on the temperature-dependent values of bulk IN625.

\section{Mesh and time step description}

The convergence study detailed in Reference 17 was used to determine the grid-independent spatial and temporal resolution for the present conditions. The study showed a grid-independent solution with a cell spacing of $12.5 \mu \mathrm{m}$ in the $x$-direction, and $6.25 \mu \mathrm{m}$ in the $y$ and $z$-directions. For the present uncertainty analysis, all simulations were conducted in a $5.0 \times 0.8 \times 0.4 \mathrm{~mm}$ $(x, y, z)$ computational domain, illustrated in Figure 1.

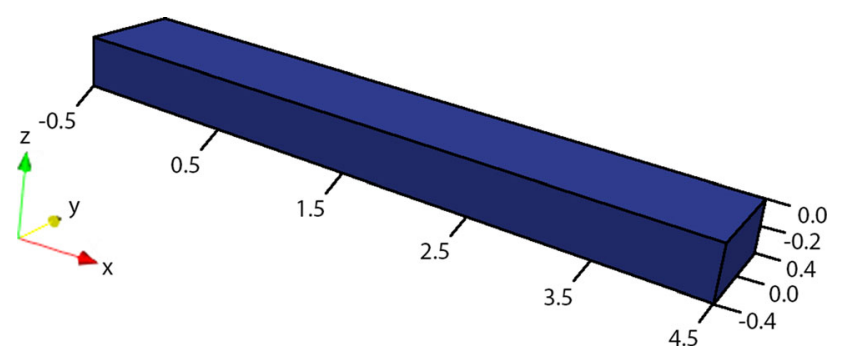

Fig. 1-Computational domain used to simulate single line scan. The scan begins with the laser spot centered at $x=0$ and traverses in the positive $x$-direction. Axes given in $\mathrm{mm}$.

A steady state condition was considered to be met when the solidification time at the surface on the melt pool centerline changed less than $1 \mu$ s from one control volume to the next. (That time is generally between $1 / 2$ and 1 pct of the solidification time.) The maximum melt pool width and depth were calculated based on the liquidus temperature at a point in the computational domain after a steady state was reached by interpolating temperature data along the $y$ and $z$-directions. Solidification time was calculated along the centerline of the melt pool surface and $15 \mu \mathrm{m}$ below the top surface by tracking the time between the liquid fraction dropping below 100 pct and reaching a value less than 0.1 pct. A timestep of $1 \mu$ s was used to ensure sufficient temporal resolution. For cases when the powder layer was simulated, it had a preset depth of $30 \mu \mathrm{m}$ where the domain below that layer used the bulk properties of IN625 to simulate a substrate.

\section{B. Uncertainty Analysis}

To analyze the propagation of input uncertainty to model outputs of interest, the numerical model was coupled with TASMANIAN,${ }^{[19-23]}$ a non-intrusive library used to construct a polynomial surrogate model. This surrogate model approximated the behavior of the numerical simulation predictions but can be sampled at a fraction of the computational expense. The replacement of the transport model with a polynomial surrogate allowed for Monte Carlo-based sampling to establish probability distribution functions (PDF) for each output of interest. The present uncertainty analysis was similar to those used in References 26, 27 applied to other solidification processes.

The upfront cost of constructing the surrogate model is dependent upon the number of points on a sparse grid, on which each point corresponds to one set of input values for the numerical model and one associated response. The number of points grows rapidly with the number of uncertain inputs. The present L-PBF model has 13 uncertain input parameters when not accounting for the powder layer, and 14 when including the powder bed due to the addition of the powder packing density. To capture the full uncertainty space, a 13 or 14-dimensional sparse grid would be needed and would require hundreds of simulations to construct the surrogate. To reduce this upfront cost, the uncertainty 
analysis in the present work was proceeded by a simple sensitivity analysis of the influence each parameters' associated uncertainty had on model predictions.

\section{Input parameters}

Density, specific heat, and thermal conductivity values for bulk IN625 were obtained from JMatPro, which unfortunately does not provide either its calculation method or the associated uncertainties. For this reason, the established uncertainties in property data were estimated based on measurement techniques for each of the properties. In addition to the uncertainty in thermophysical properties, several other model parameters possess a degree of uncertainty due to variation in material and/or measurement procedure. Uncertainty in laser power may arise due to noise and variations in current provided to the pumping source. As discussed in Reference 17, the penetration depth $(d)$ in the present model was calibrated to ensure numerical stability while sufficient energy is imparted. A large uncertainty of 5.0 microns was used here to account for the deviation from the true physics of the laser-metal interactions. Work by Deisenroth et al. ${ }^{[2]}$ found that even under favorable conditions, uncertainty in measured absorbed energy values is in excess of 10 pct. In the present model, the laser absorptivity parameter was not determined directly from experimental measurements, but rather by model calibration by comparing measured and predicted temperatures and to fit melt pool dimensions. The laser absorptivity uncertainty value was estimated to be 15 pet of the baseline value of 0.31 here. Finally, powder packing density was assigned an uncertainty of $0.05(\approx 8$ pct) to account for local variations in packing density arising from variations in particle size. All uncertainties were assumed to follow a Gaussian distribution and the uncertainty values given in Table I are representative of three standard deviations $(3 \sigma)$, a 99 pct confidence interval.

\section{Sensitivity analysis}

The numerical model contains 13 or 14 input parameters, depending on whether a powder layer is included, all of which have an associated uncertainty. Accounting for all these inputs in the uncertainty propagation study would be computationally expensive due to the high dimensionality of the uncertainty space. In an effort to reduce the number of dimensions, a sensitivity analysis was carried out. For each uncertain input, outputs were calculated using the numerical model at the mean input value and at the two extremes of the range of uncertainty; these three evaluations give a simple measure of the effect of input uncertainty on the output uncertainty.

The criteria used to determine whether the uncertainty for a particular input parameter is significant are:

- Change in solidification time is more than 1 pct of the baseline values;

- Change in melt pool width is more than 1 pct of the baseline width;

- Change in melt pool depth is more than 1 pct of the baseline depth.
Input parameters that met any of these three criteria were included in the subsequent uncertainty analysis as discussed in Section III-C.

\section{Constructing and testing the surrogate model}

A sparse grid containing the input parameters that were not down-selected by the preliminary sensitivity analysis was generated for the model with and without a powder layer. The sparse grids were generated in TASMANIAN using Clenshaw-Curtis quadrature rules and Lagrangian polynomials were used to interpolate between points when constructing the surrogate. These methods were used as they are computational cheap (few grid points) and the model behavior is expected to be smooth throughout the uncertainty space. A level-two analysis was used to keep the number of grid points, and thus the number of numerical simulations needed, relatively low.

Each point on the sparse grid represents a set of input parameter values that is passed through the numerical model. A polynomial response surface was formed by interpolating among all the response values. This response surface acts as a surrogate model which can then be interrogated rapidly for the outputs of interest. This process was repeated for all the outputs to generate a surrogate model for each.

To validate that the surrogate model accurately represented the behavior of the simulation, the surrogate was sampled at values away from the grid points used to construct it where the predictions are the least reliable, and compared to the predictions at those points from the numerical model. The comparison of the outputs of the surrogate to the numerical model with the full physics ensures that behavior is correctly captured by the interpolated polynomial over the entire input range. Results for the surrogate validation showed solidification times and pool dimensions within 3 pet of the numerically calculated value.

\section{Monte Carlo sampling}

Once validated, the surrogate model was sampled with confidence that the predictions were representative of the transport model. This is beneficial as the polynomial response surface can be sampled at a fraction of the cost of the numerical model. To produce probability distribution functions (PDFs) for each of the outputs of interest, the surrogates for both powder bed representations were sampled over the uncertainty ranges using a Monte Carlo-based method. To ensure a statistically valid representation of the models, 10 million random values for each input parameter were selected from the Gaussian distribution around its most likely value. The pseudorandom number generating algorithm, Mersenne Twister, was utilized to carry out the Monte Carlo random sampling.

\section{Reduced-Order Uncertainty Analysis}

In addition to the full uncertainty propagation analysis which included all inputs to which output uncertainties were sufficiently sensitive, as defined in Section II-B-B, a reduced-order analysis was also 
performed which only accounted for the three parameters which caused the largest output uncertainty in each of the powder bed models. This guarantees that only 19 numerical simulations were needed in constructing the polynomial surrogate model which is a drastic reduction in the computational expense need to account for all input parameter uncertainties, while still capturing the majority of the uncertainty in model predictions.

\section{RESULTS}

\section{A. Baseline}

Baseline model predictions for the two powder bed models were established using the mean values listed in Table I. As illustrated by Figure 2, the temperature rise at the edge of the numerical domain is negligible, showing that the size of the domain was sufficient to model a single pass in a semi-infinite space. Table III presents solidification time data at several points along the middle of the domain illustrating a steady state was achieved at the point of measurement.

\section{B. Parameter Sensitivity Analysis}

A simple analysis was conducted to determine the uncertainty of which input parameters had an appreciable effect on model prediction reliability (Section II-B-B). Each parameter was individually adjusted $\pm 3 \sigma$ from its mean value in Table I to capture the extremes of the uncertainty bounds. Adjusted parameters which caused a change in solidification time greater than 1 pet and/or a change in melt pool dimensions greater than 1 pct of the baseline were
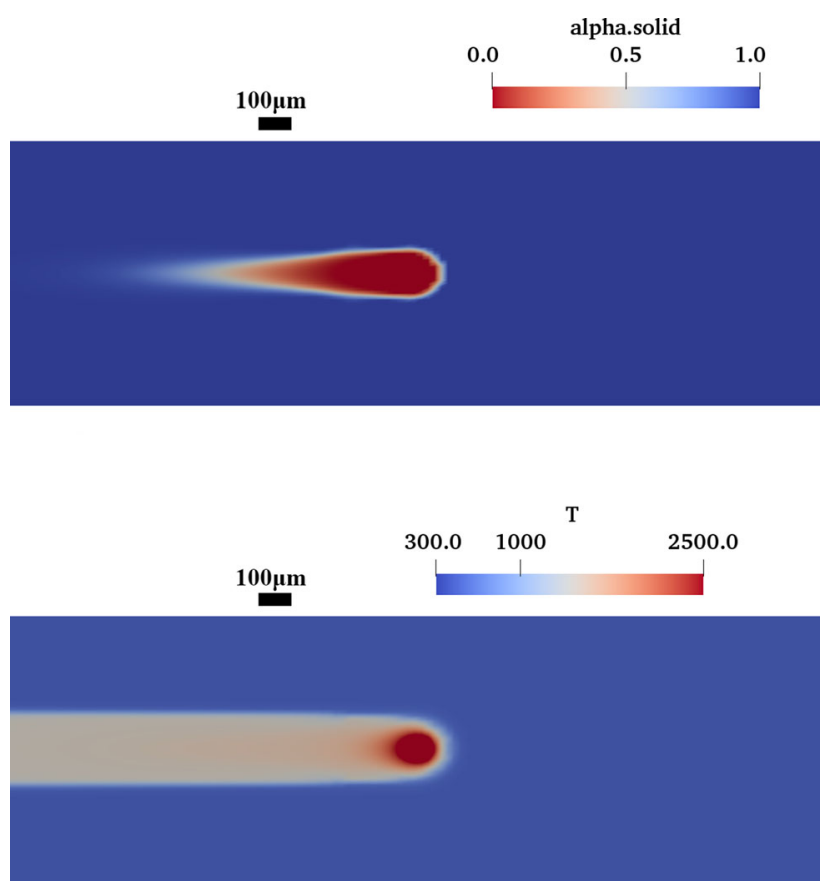

Fig. 2-Top-down view of single pass simulation for solid fraction and temperature $(\mathrm{K})$. considered 'high-sensitivity' parameters and included in the full uncertainty quantification analysis. Figures 3 and 4 summarize the analysis for the laser powder bed fusion process for the cases when the powder bed is not included for and when it is modeled as a mixture of metal particles and a cover gas, respectively. Figure 3 shows that the five parameters to which model prediction uncertainty without a powder layer was found to have a high-sensitivity are (in order of importance) laser absorption, the specific heat of solid and liquid phases of IN625, laser power, and laser penetration depth. When a powder layer is modeled, the inputs passing the criteria in Section II-B-B are the same, but also include the powder packing density as shown by Figure 4 .

\section{Uncertainty Analysis}

\section{Model without powder layer}

To analyze the total uncertainty in the model predictions, a level-two analysis of the five significant parameters from Figure 3 was conducted. To build the surrogate model, a five-dimensional sparse grid with 51 total points was developed using TASMANIAN to represent the parameter space inside the ranges of the input uncertainties. The input values corresponding to each these points were fed into the OpenFOAM model, the simulations were performed, and the outputs were fed back as a response to the sparse grid. Using these 51 input grid points and the associated outputs, the surrogate model for each of the outputs of interest was constructed.

Before sampling, the surrogate model for each output of interest was validated at several points not located at grid points. This ensures that the quadrature rules used in constructing the sparse grid and the interpolation scheme between the grid points are valid. Additionally, this ensures the level of analysis was high enough to accurately represent the behavior of the numerical model. The grid points on the sparse grid occur at \pm $3 \sigma$ for each parameter. The points used for validation occur at $-\sigma,-2 \sigma, \sigma$, and $2 \sigma$ for each of the input parameters. These check points are provided in Table IV, as are the outputs of interest generated by the full numerical L-PBF model and the surrogates, both with and without the powder layer.

Table IV shows that the surrogate models accurately represent the transport model for predictions of solidification time and melt pool dimensions; the solidification times and pool dimensions are within 3 pet of the full numerical model results. These surrogate models were then sampled 10 million times to produce the probability distribution functions shown in Figure 5. Accompanying statistics for the Monte Carlo sampling are detailed in Table V.

\section{Model with Powder Layer}

The model with a powder layer was treated similarly. In this case, six parameters were identified as high-sensitivity; thus, a six-dimensional sparse grid was generated. This level-two sparse grid contained 73 total points, requiring 73 evaluations of the numerical model to develop the surrogate. Validation data for the 
Table III. Comparison of Steady State Solidification Times With and Without a Simulated Powder Layer Showing a Steady State Was Reached

\begin{tabular}{lcr}
\hline Probe Location $(x, y, z)(\mathrm{mm})$ & $t_{\mathrm{s}}(\mathrm{ms})($ No Powder) & $t_{\mathrm{s}}(\mathrm{ms})$ (Simulated Powder Layer) \\
\hline$(2.00,0.0,-0.015)$ & 0.148 & 0.186 \\
$(2.25,0.0,-0.015)$ & 0.148 & 0.186 \\
$(2.50,0.0,-0.015)$ & 0.148 & 0.186 \\
$(2.75,0.0,-0.015)$ & 0.148 & 0.186 \\
$(3.00,0.0,-0.015)$ & 0.148 & 0.186 \\
\hline
\end{tabular}

Times were measured along the scan direction, at the scan centerline along the top surface.

\section{Sensitivity - No Powder}

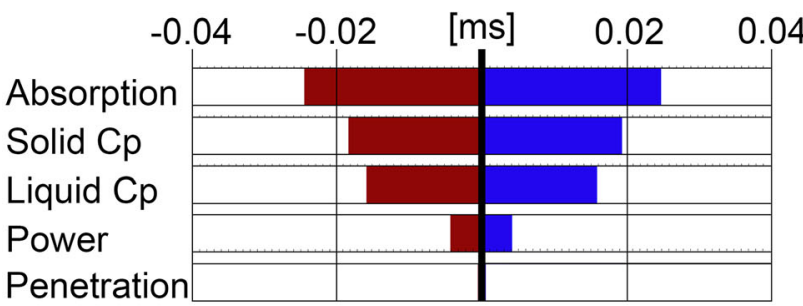

(a)

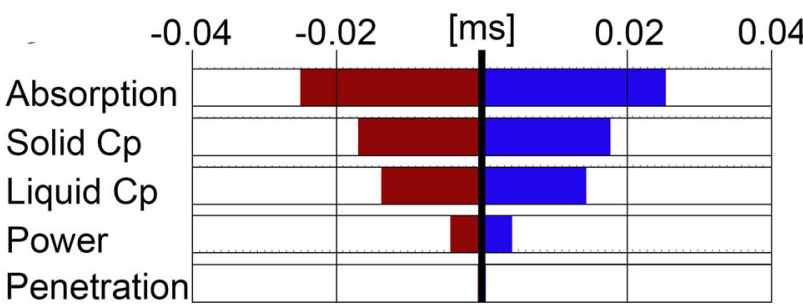

(b)

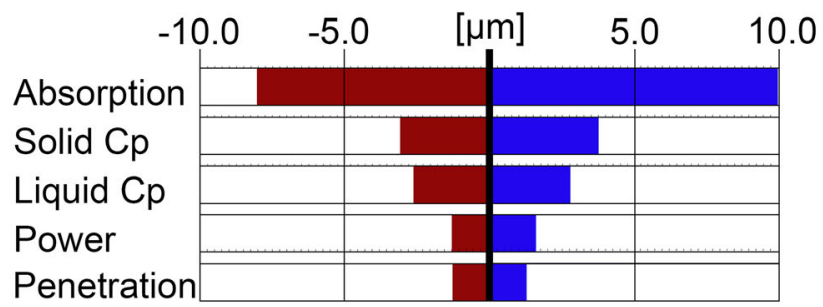

(c)

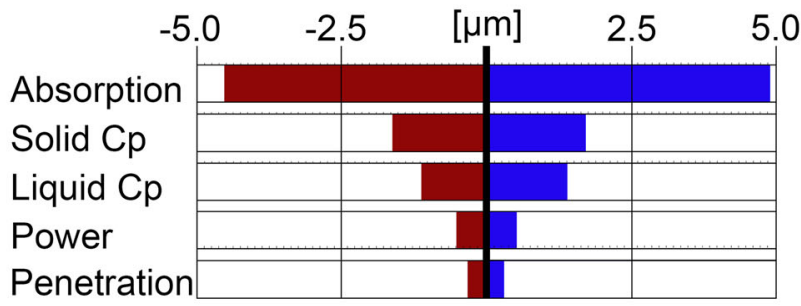

(d)

Fig. 3- Sensitivity analysis for solidification time at and below the top surface $(a, b),(c)$ melt pool width, and $(d)$ melt pool depth for cases without a simulated powder layer.

surrogate models is provided in Table IV. Again, the surrogate models accurately represents the transport model and is used for Monte Carlo sampling with 10 million samples of each surrogate. The generated probability distribution functions are shown in Figure 6 and sampling statistics are provided in Table V

\section{Reduced-Order Uncertainty Analysis}

As many of the model input parameters uncertainties had little effect on uncertainty in the model predictions, the crude sensitivity analysis reduced the dimension of the uncertainty space from 13 to 5 when not accounting for the powder bed, and from 14 to 6 for the mixture powder bed model. Despite this reduction, to construct the second order polynomial surrogate models, 51 and 73 numerical simulations were required, respectively. As the present work has assumed the processing conditions would result in conduction dominant heat transfer, fluid mechanics has been neglected, reducing the number of uncertain input parameters while also drastically speeding up the computations. As a result, the required numerical simulations can be performed relatively quickly, on the order of 100 clock hours using 128 total cores across two AMD Epyc 7662 "Rome" processors; however, if fluid mechanics were included this increases by an order of magnitude. Additionally, including the fluid mechanics incorporates several other input parameters whose uncertainty must be accounted for (e.g., surface tension gradient). Furthermore, the present analysis is only level-two. The comparison of surrogate model predictions to data obtained from the transport model presented in Tables IV show that this low level of analysis is sufficient to produce a reliable surrogate for the present study, but this may not hold true when incorporating fluid flow. Higher-order analysis would require more points on the sparse grid and thus more numerical simulations to generate an accurate surrogate.

In addition to this uncertainty analysis including 5 or 6 parameters deemed to be high-sensitivity, a reduced-order analysis was conducted with only the three most significant parameters. The reduced-order analysis follows the same process of generating a sparse grid and a surrogate model. Likewise, the 3 parameter surrogate model was also validated at values away from grid points to ensure it accurately represented the numerical 


\section{Sensitivity - Simulated Powder}

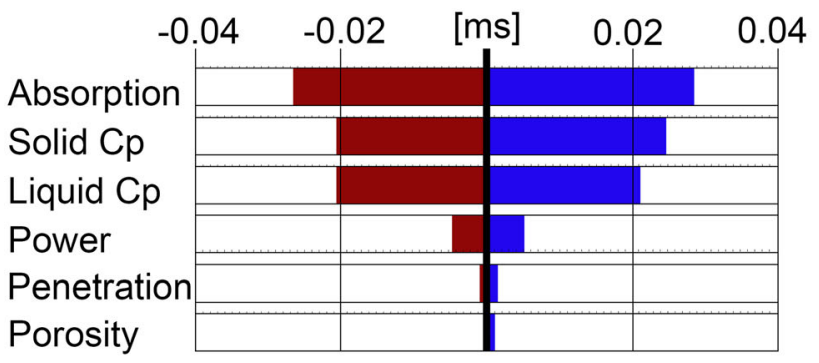

(a)

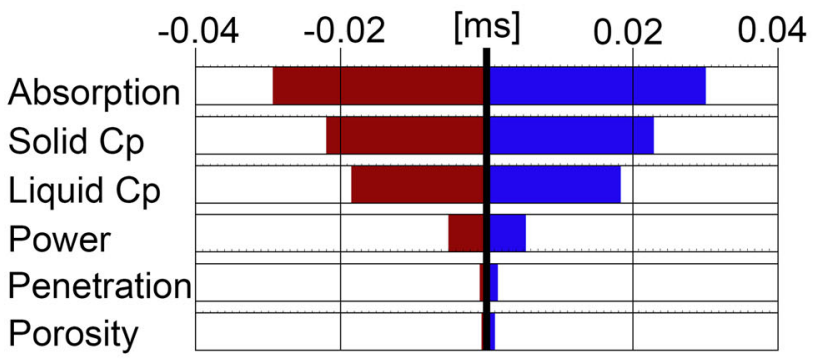

(b)

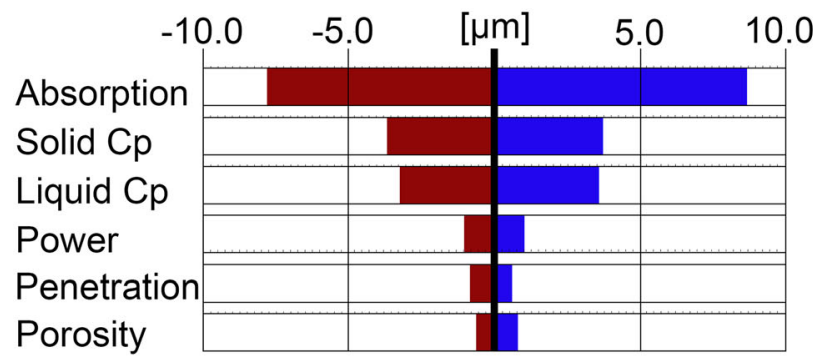

(c)

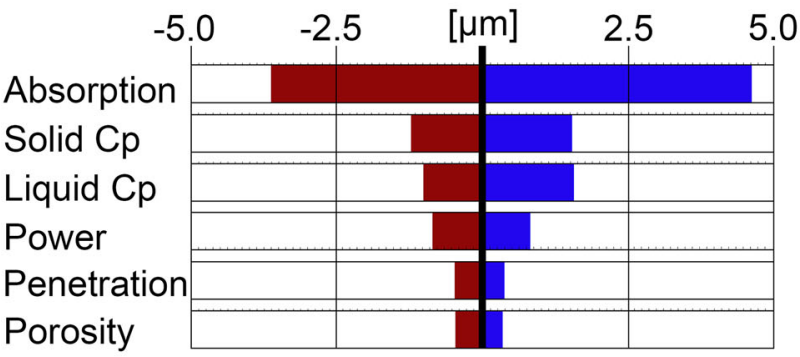

(d)

Fig. 4 - Sensitivity analysis for solidification time at and below the top surface $(a, b),(c)$ melt pool width, and $(d)$ melt pool depth for cases with a simulated powder layer.

Table IV. Numerical Model Outputs of Interest at Parameter Values Away From Sparse Grid Points, Compared to Surrogate Model Predictions for Cases With and Without the Powder Layer

\begin{tabular}{|c|c|c|c|c|c|}
\hline \multirow[b]{2}{*}{ Inputs } & \multirow[b]{2}{*}{ Output } & \multicolumn{2}{|c|}{ No Powder } & \multicolumn{2}{|c|}{ Simulated Powder } \\
\hline & & OpenFOAM & Surrogate & OpenFOAM & Surrogate \\
\hline$P=193.5 \mathrm{~W}$ & $t_{\mathrm{s}}(\mathrm{ms})($ surface $)$ & 0.139 & 0.139 & 0.178 & 0.178 \\
\hline$\eta=0.295$ & $t_{\mathrm{s}}(\mathrm{ms})($ sub-surface $)$ & 0.134 & 0.134 & 0.167 & 0.166 \\
\hline$d=18.67 \mu \mathrm{m}$ & width $(\mu \mathrm{m})$ & 122.8 & 123.1 & 141.3 & 141.0 \\
\hline$\phi=0.583$ & depth $(\mu \mathrm{m})$ & 35.2 & 34.8 & 35.5 & 36.0 \\
\hline \multicolumn{6}{|l|}{$c_{\mathrm{ns}}=c_{\mathrm{nl}}=744.7 \mathrm{~J} / \mathrm{kgK}$} \\
\hline$P=192.0 \mathrm{~W}$ & $t_{\mathrm{s}}(\mathrm{ms})($ surface $)$ & 0.131 & 0.132 & 0.168 & 0.168 \\
\hline$\eta=0.280$ & $t_{\mathrm{s}}(\mathrm{ms})$ (sub-surface) & 0.125 & 0.125 & 0.157 & 0.157 \\
\hline$\phi=0.566$ & width $(\mu \mathrm{m})$ & 120.4 & 119.6 & 139.4 & 140.2 \\
\hline$d=17.33 \mu \mathrm{m}$ & depth $(\mu \mathrm{m})$ & 33.8 & 33.4 & 34.3 & 34.4 \\
\hline \multicolumn{6}{|l|}{$c_{\mathrm{ps}}=c_{\mathrm{pl}}=734.6 \mathrm{~J} / \mathrm{kgK}$} \\
\hline$P=196.5 \mathrm{~W}$ & $t_{\mathrm{s}}(\mathrm{ms})($ surface $)$ & 0.156 & 0.157 & 0.197 & 0.196 \\
\hline$\eta=0.325$ & $t_{\mathrm{s}}(\mathrm{ms})($ sub-surface $)$ & 0.151 & 0.151 & 0.188 & 0.187 \\
\hline$\phi=0.617$ & width $(\mu \mathrm{m})$ & 127.5 & 127.0 & 145.2 & 144.6 \\
\hline$d=21.33 \mu \mathrm{m}$ & depth $(\mu \mathrm{m})$ & 38.6 & 38.1 & 39.0 & 38.7 \\
\hline \multicolumn{6}{|l|}{$c_{\mathrm{ps}}=c_{\mathrm{pl}}=764.8 \mathrm{~J} / \mathrm{kgK}$} \\
\hline$P=198.0 \mathrm{~W}$ & $t_{\mathrm{s}}(\mathrm{ms})($ surface $)$ & 0.165 & 0.165 & 0.207 & 0.202 \\
\hline$\eta=0.34$ & $t_{\mathrm{s}}(\mathrm{ms})$ (sub-surface) & 0.160 & 0.160 & 0.198 & 0.197 \\
\hline$\phi=0.633$ & width $(\mu \mathrm{m})$ & 129.0 & 128.9 & 146.5 & 146.2 \\
\hline$d=23.67 \mu \mathrm{m}$ & depth $(\mu \mathrm{m})$ & 40.0 & 40.5 & 40.2 & 41.1 \\
\hline$c_{\mathrm{ps}}=c_{\mathrm{pl}}=774.9 \mathrm{~J} / \mathrm{kgK}$ & & & & & \\
\hline
\end{tabular}

model. By reducing the dimensionality of the uncertainty space from 5 and 6 for the two powder bed models down to only 3 , the total number of numerical simulations required to construct the surrogate model is reduced to 19. Thus, computational expense is further reduced. The motivation being that if we can show this analysis is sufficient to characterize the reliability of the model outputs for the conduction-dominated regime, then we have an easier starting point for a later, more comprehensive analysis including fluid flow. 


\section{Full Analysis - No Powder}

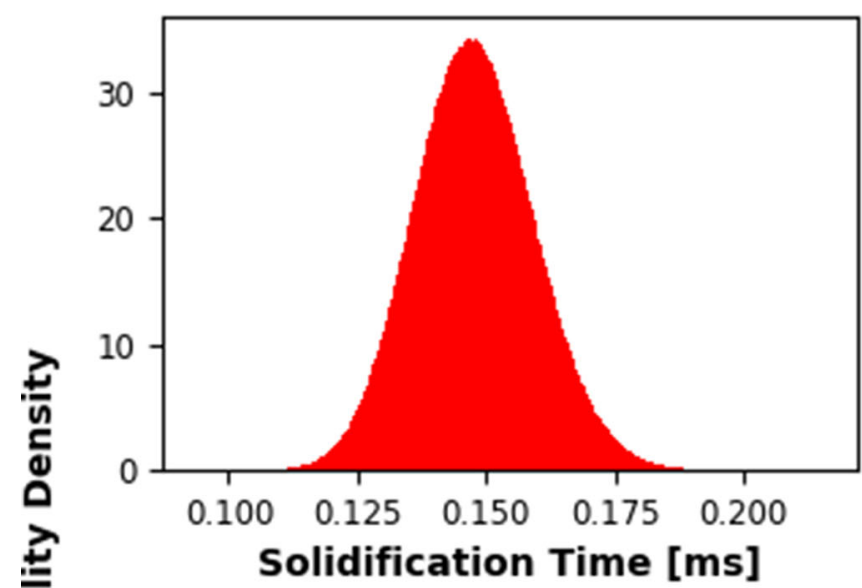

(a)

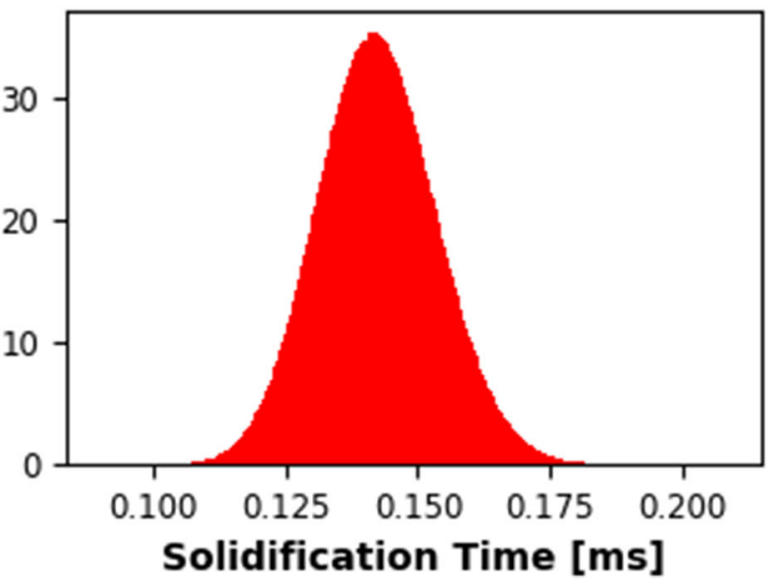

(b)

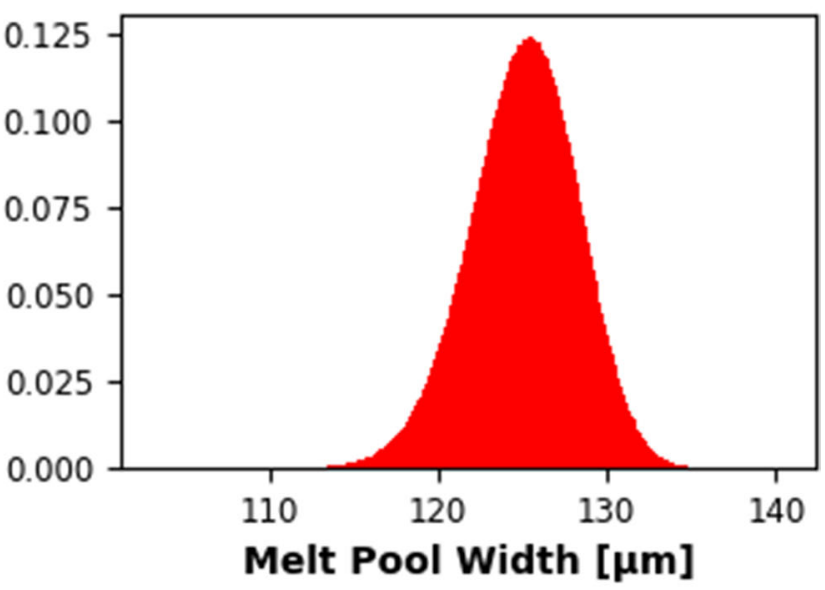

(c)

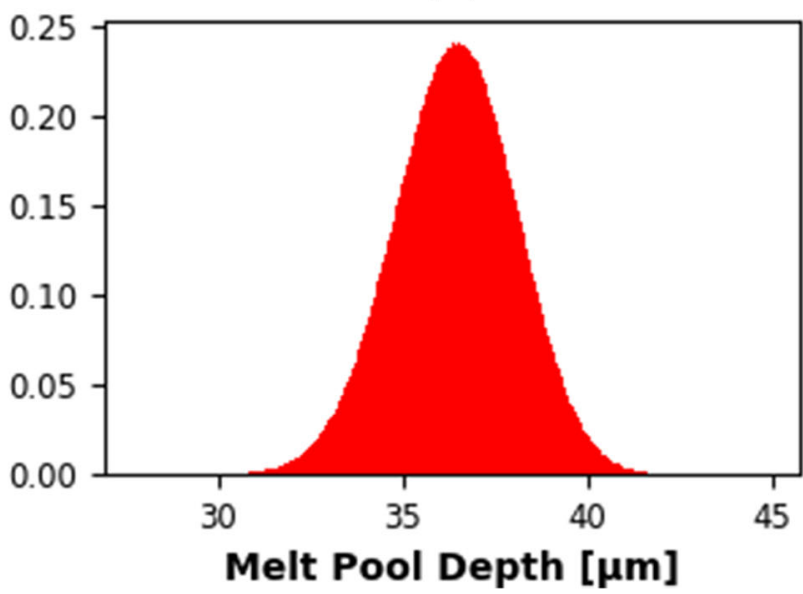

(d)

Fig. 5-Probability distribution functions of the solidification time at the surface $(a)$ and sub-surface $(b)$, and melt pool dimensions $(c, d)$ generated by sampling the polynomial surrogate model 10 million times.

1. Reduced-order: model without powder layer

Based on the sensitivity analysis and the results presented in Figure 3, the three parameters deemed most significant when the powder bed is not modeled were laser absorption and the solid and liquid specific heats. A 3-dimensional, level-2 sparse grid was generated for these three uncertain input parameters. This sparse grid, shown in Figure 7, has only 19 points requiring full numerical simulations.

Once the surrogate model was constructed, it was validated as before. Results for the validation are presented in Table VI. After validation that the reduced-order surrogate accurately represented the transport model under the same conditions, the surrogate was sampled 10 million times for each of the outputs of interest, generating output probability distribution functions. These PDFs are shown in Figure 8 and sampling statistics are displayed in Table VII.

\section{Model with powder layer}

From the sensitivity analysis and Figure 4, the three most sensitive parameters for the mixed properties powder bed model were also laser absorption, and solid and liquid specific heat. The three dimensional, level-2 sparse grid for these input parameters is the same as before as shown in Figure 7. Validation of the constructed surrogate is tabulated in Table VI. and the produced probability distribution functions are illustrated in Figure 9. Statistics for the Monte Carlo sampling can be found in Table VII.

\section{E. Comparison of Reduced-Order to Full Analysis}

The purpose of the reduced-order analysis was to decrease as much as reasonable the dimensionality of the uncertainty space for models of the L-PBF process, so as to minimize the computational expense without a 
Table V. Statistics for the Monte Carlo Sampling of the Full Surrogate Model Predictions With and Without the Simulated Powder Layer

Full Analysis Statistics

\begin{tabular}{|c|c|c|c|c|}
\hline & \multicolumn{2}{|c|}{ No Powder } & \multicolumn{2}{|c|}{ Powder } \\
\hline & Mean & $\sigma$ & Mean & $\sigma$ \\
\hline$t_{\mathrm{s}}(\mathrm{ms})($ Surface $)$ & 0.148 & $1.17 \times 10^{-2}$ & 0.186 & $1.47 \times 10^{-2}$ \\
\hline$t_{\mathrm{s}}(\mathrm{ms})($ Sub-Surface $)$ & 0.142 & $1.13 \times 10^{-2}$ & 0.177 & $1.40 \times 10^{-2}$ \\
\hline Melt Pool Width $(\mu \mathrm{m})$ & 125.1 & 3.23 & 142.7 & 2.89 \\
\hline Melt Pool Depth $(\mu \mathrm{m})$ & 36.4 & 1.65 & 37.3 & 1.53 \\
\hline
\end{tabular}

\section{Full Analysis - Simulated Powder}

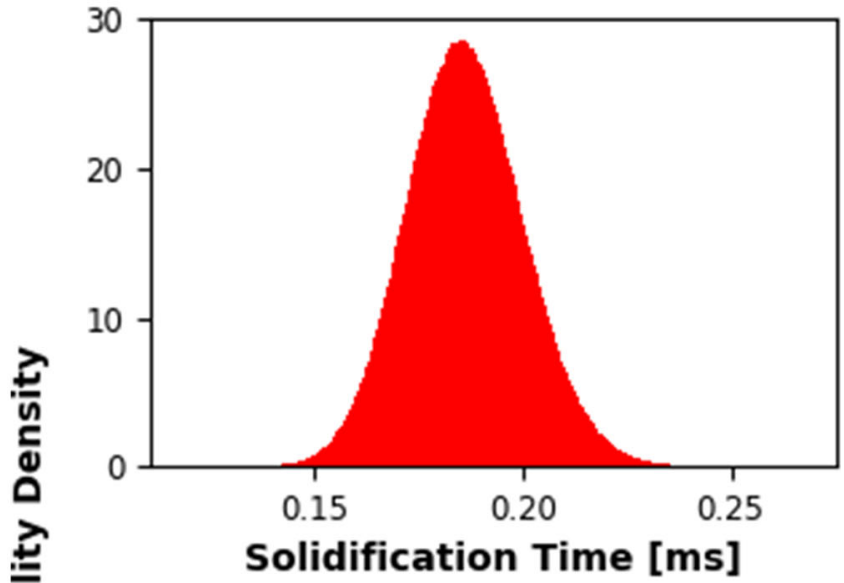

(a)

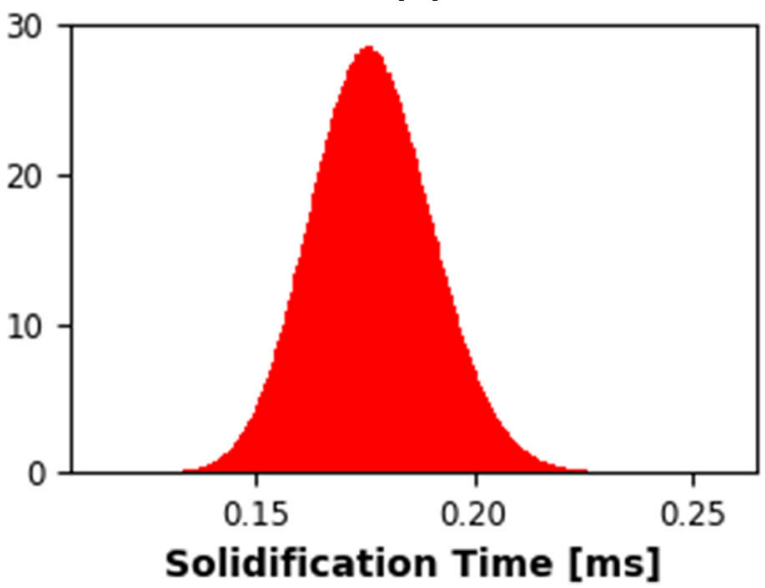

(b)

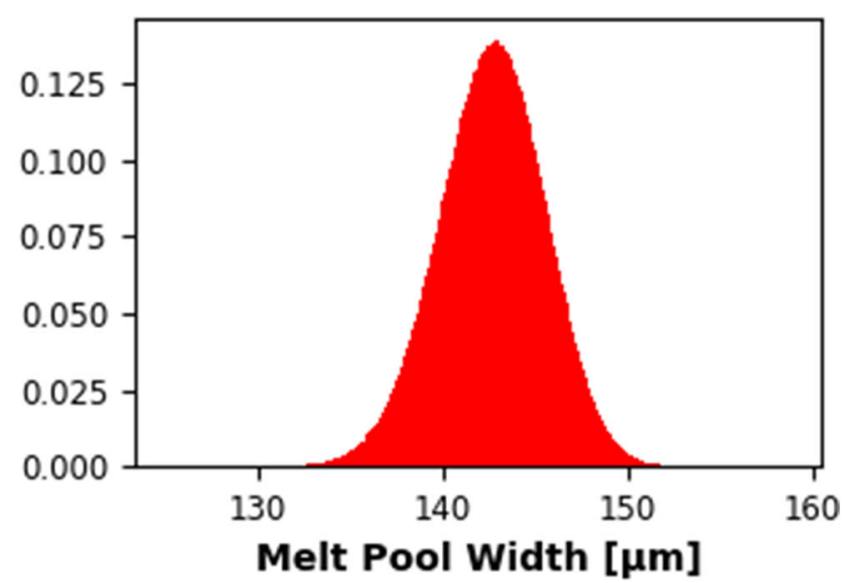

(c)

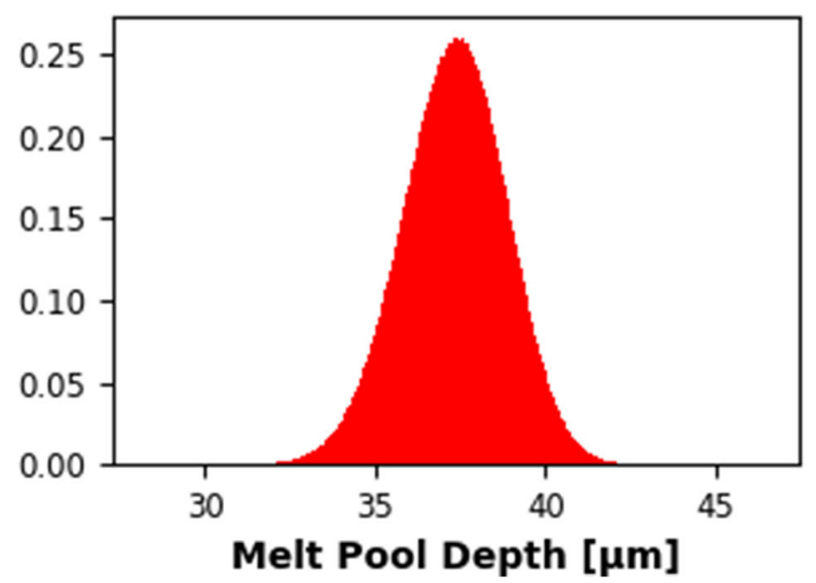

(d)

Fig. 6-Probability distribution functions of the solidification time at the surface $(a)$ and sub-surface $(b)$, and melt pool dimensions $(c, d)$ generated by sampling the polynomial surrogate model 10 million times.

significant loss in detail. The probability distribution functions produced by the reduced-order analysis compared to those of the full analysis, including all parameters that were deemed high-sensitivity are shown in Figures 10 and 11 showing good agreement between the two with only slight narrowing of the reduced-order analysis. A comparison of the sampling statistics for the full analysis vs the reduced-order is provided in Table VIII showing similar mean values with a small reduction in standard deviation. 


\section{DISCUSSION}

The results in Section III show that for the numerical model of L-PBF, with or without the inclusion of a powder bed, the uncertainty in the predictions arose almost entirely from uncertainty in the laser absorptivity and the specific heats of the solid and liquid phases of IN625. The output uncertainties were almost entirely insensitive to most of the other input parameter uncertainties in Table I. Comparisons between probability density functions (Figures 10 and 11) and sampling statistics (Table VIII) obtained from the reduced-order analysis and the more comprehensive analyses showed only slight shifts in means and minimal broadening of the output distributions for both powder bed representations. For many parameters, this lack of sensitivity can be explained by looking at the low uncertainty in input values. For example, density and thermal conductivity are properties with small measurement variability, so their uncertainty is small. While the model predictions themselves may be highly sensitive to these thermophysical properties, the uncertainty in these properties is small and so contribute little to output uncertainty.

Uncertainty in laser absorptivity was found to have the largest contribution to the uncertainty in the outputs of interest for both representations of the powder bed. As discussed in Reference 17, the laser absorptivity value used in the transport model is not based on experimental measurements, rather on the results produced when different values were calibrated to experimental measurements of melt pool dimensions and temperature, but there is a high uncertainty in that fit. Additionally, this parameter is present in the volumetric heat source term, Eq. [3]. This term controls the amount of thermal energy imparted on the system and the effects of that transfer are highly sensitive to variation in laser absorption and its uncertainty. The specific heats of the

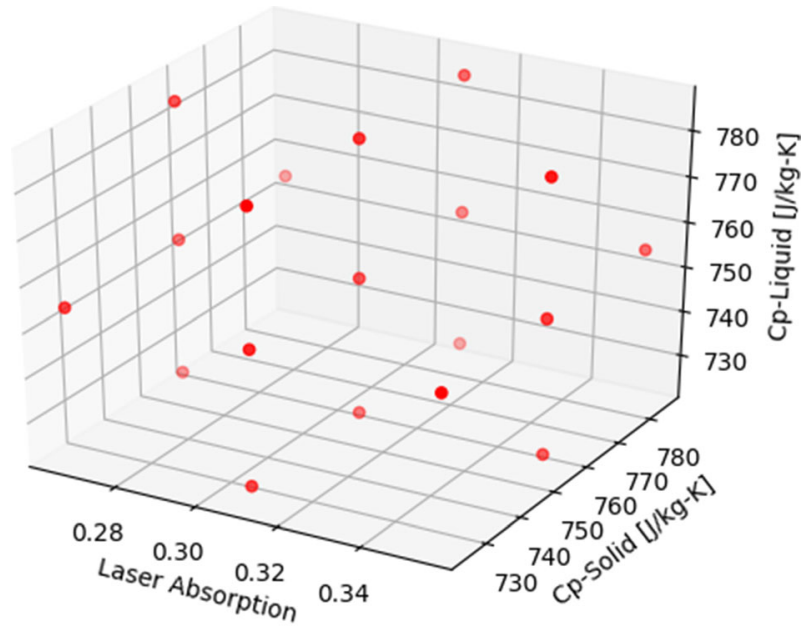

Fig. 7-Three dimensional, level-2 sparse grid for the reduced-order analysis for both powder models, constructed using TASMANIAN. liquid and solid phases are the other two most important contributions to the output uncertainties. These properties control the uncertainty in the metal thermal capacity, $\rho \mathrm{c}_{\mathrm{p}}$, which in turn controls the cooling rate. As mentioned before, the uncertainty in density is low thus which is why its' contribution to model prediction uncertainty is much smaller than in the specific heat despite being multiplied in all occurrences in the energy conservation equation.

Additionally, when comparing the results of the two different approaches (with and without the powder layer), it is seen that the solidification times at positions on and $15 \mu \mathrm{m}$ below the top surface are significantly different. This deviation can be attributed to the much lower thermal diffusivity of the powder bed. The heat conducts more slowly outward from the sides of the melt pool into the unmelted powder and the majority of thermal energy conducts away from the melt pool occurs down into the substrate. Not able to shed heat laterally as fast as into a consolidated metal, the melt pool solidification rate is greatly reduced.

Finally, the observation that a reduced-order analysis captures the vast majority of input parameter contribution to the model predictions' uncertainty is important, because reducing the dimensions of the uncertainty space reduces the number of expensive simulations required to construct a surrogate model for sampling. The reduction to only three important parameters (instead of 13 or 14) will enable faster analysis of similar problems such as processing conditions with higher Péclet numbers where fluid flow must be considered, and with it, several new uncertain parameters such as the surface tension gradient.

\section{CONCLUSION}

This work used uncertainty quantification techniques to analyze a finite volume model for the L-PBF process, and to determine the most significant sources of input uncertainty and their effect on model prediction accuracy. Preliminary results show the propagation of input uncertainties to model predictions of melt pool dimensions and solidification time for a single-pass simulation in the conduction dominant regime.

Uncertainty in laser absorption and the metal specific heats were found to have the largest effect on the reliability of predictions of the L-PBF model with or without the use of a powder bed. Comparing the PDFs for the reduced-order uncertainty analysis for these three processes to the full comprehensive analysis, which included the uncertainty in laser power, numerical penetration depth, and packing density of the powder bed, show that the three variables mentioned above dominate the uncertainty in model predictions. Accounting for only these three variables significantly reduces computational time while still providing accurate measurements of model output uncertainty. 
Table VI. Numerical Model Predictions of Outputs of Interest at Parameter Values Away From Points on the Sparse Grid Compared to the Reduced-Order Surrogate Model Predictions at Those Values When the Powder Bed is not Included

\begin{tabular}{|c|c|c|c|c|c|}
\hline \multirow[b]{2}{*}{ Inputs } & \multirow[b]{2}{*}{ Output } & \multicolumn{2}{|c|}{ No Powder } & \multicolumn{2}{|c|}{ Simulated Powder } \\
\hline & & OpenFOAM & Surrogate & OpenFOAM & Surrogate \\
\hline$\eta=0.295$ & $t_{\mathrm{s}}(\mathrm{ms})($ surface $)$ & 0.141 & 0.141 & 0.180 & 0.178 \\
\hline \multirow[t]{3}{*}{$c_{\mathrm{ps}}=c_{\mathrm{pl}}=744.7 \mathrm{~J} / \mathrm{kgK}$} & $t_{\mathrm{s}}(\mathrm{ms})($ sub-surface $)$ & 0.135 & 0.135 & 0.170 & 0.168 \\
\hline & width $(\mu \mathrm{m})$ & 122.5 & 122.6 & 140.6 & 140.3 \\
\hline & depth $(\mu \mathrm{m})$ & 35.4 & 35.2 & 35.8 & 36.5 \\
\hline$\eta=0.280$ & $t_{\mathrm{s}}(\mathrm{ms})($ surface $)$ & 0.133 & 0.133 & 0.171 & 0.171 \\
\hline \multirow[t]{3}{*}{$c_{\mathrm{ps}}=c_{\mathrm{pl}}=734.6 \mathrm{~J} / \mathrm{kgK}$} & $t_{\mathrm{s}}(\mathrm{ms})($ sub-surface $)$ & 0.128 & 0.128 & 0.160 & 0.160 \\
\hline & width $(\mu \mathrm{m})$ & 119.9 & 119.9 & 138.7 & 138.1 \\
\hline & depth $(\mu \mathrm{m})$ & 34.4 & 34.1 & 34.9 & 35.1 \\
\hline$\eta=0.325$ & $t_{\mathrm{s}}(\mathrm{ms})($ surface $)$ & 0.155 & 0.155 & 0.195 & 0.195 \\
\hline \multirow{3}{*}{$c_{\mathrm{ps}}=c_{\mathrm{pl}}=764.8 \mathrm{~J} / \mathrm{kgK}$} & $t_{\mathrm{s}}(\mathrm{ms})$ (sub-surface) & 0.150 & 0.150 & 0.186 & 0.185 \\
\hline & width $(\mu \mathrm{m})$ & 127.8 & 127.5 & 145.7 & 144.9 \\
\hline & depth $(\mu \mathrm{m})$ & 38.3 & 37.6 & 38.8 & 38.3 \\
\hline$\eta=0.34$ & $t_{\mathrm{s}}(\mathrm{ms})($ surface $)$ & 0.162 & 0.162 & 0.203 & 0.203 \\
\hline \multirow{3}{*}{$c_{\mathrm{ps}}=c_{\mathrm{pl}}=774.9 \mathrm{~J} / \mathrm{kgK}$} & $t_{\mathrm{s}}(\mathrm{ms})$ (sub-surface) & 0.157 & 0.157 & 0.194 & 0.194 \\
\hline & width $(\mu \mathrm{m})$ & 129.9 & 129.7 & 147.4 & 146.7 \\
\hline & depth $(\mu \mathrm{m})$ & 39.3 & 38.9 & 39.6 & 39.2 \\
\hline
\end{tabular}

\section{Reduced Order - No Powder}

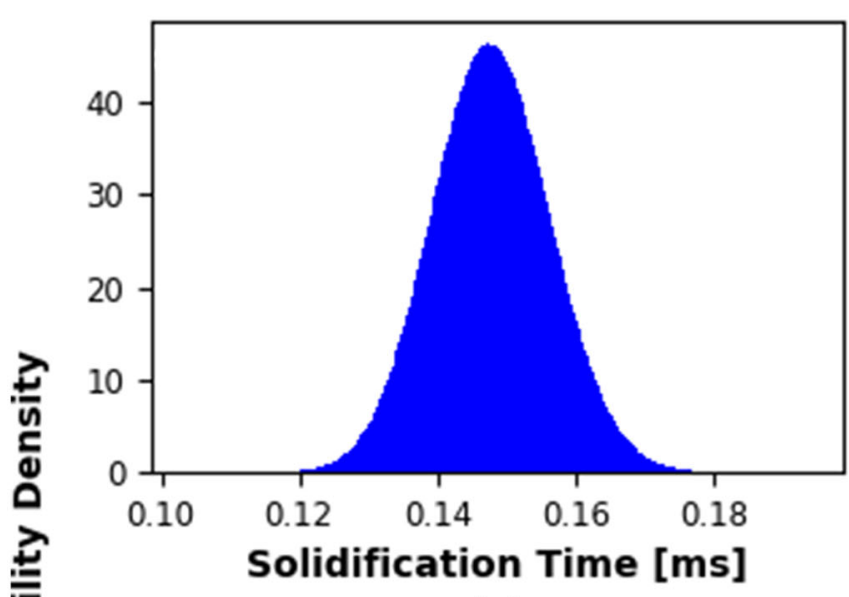

(a)

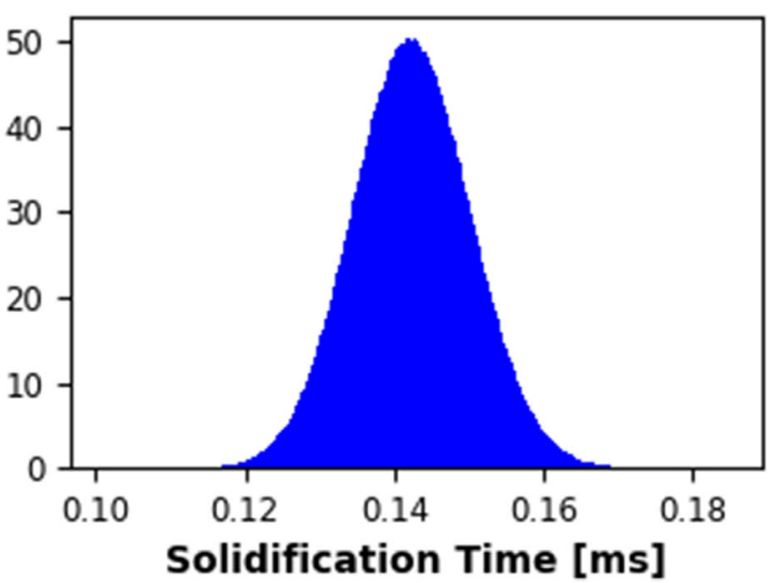

(b)

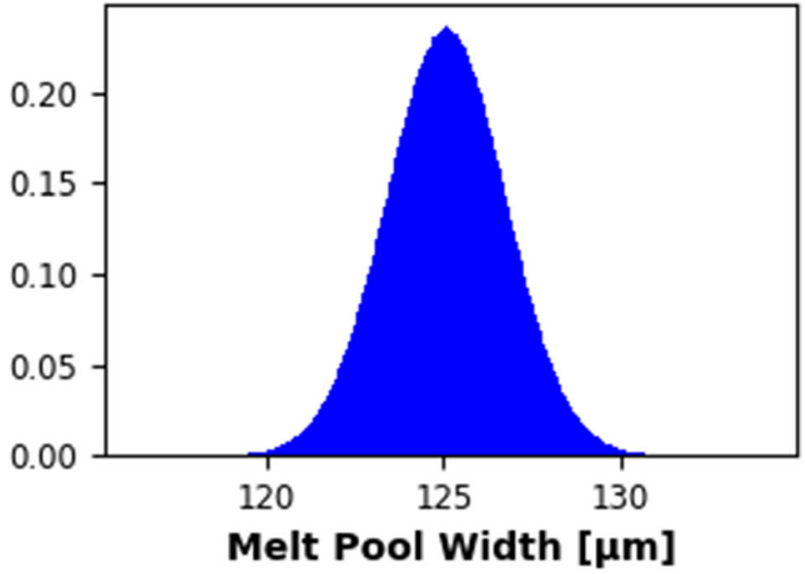

(c)

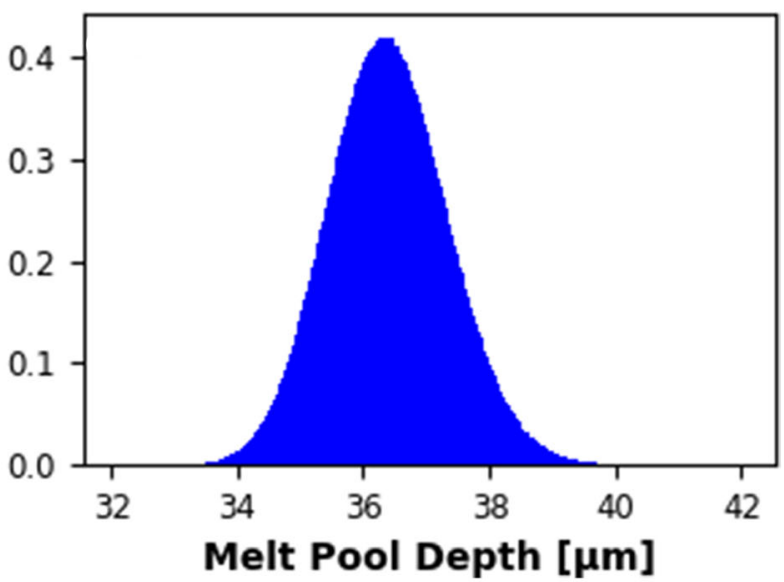

(d)

Fig. 8-Probability distribution functions of the solidification time at the surface $(a)$ and sub-surface $(b)$, and melt pool dimensions $(c, d)$ for the reduced-order analysis when the powder layer is not included. 
Table VII. Statistics for the Monte Carlo Sampling of the Analysis Surrogate Model Predictions of Solidification Time on and 15 $\mu \mathrm{m}$ Below the Top Surface, and Melt Pool Dimensions With and Without the Powder Layer

\begin{tabular}{|c|c|c|c|c|}
\hline \multicolumn{5}{|c|}{ Reduced-Order Analysis Statistics } \\
\hline & \multicolumn{2}{|c|}{ No Powder } & \multicolumn{2}{|c|}{ Powder } \\
\hline & Mean & $\sigma$ & Mean & $\sigma$ \\
\hline$t_{\mathrm{s}}(\mathrm{ms})($ Surface $)$ & 0.148 & $0.86 \times 10^{-2}$ & 0.186 & $1.08 \times 10^{-2}$ \\
\hline$t_{\mathrm{s}}(\mathrm{ms})($ Sub-surface $)$ & 0.142 & $0.79 \times 10^{-2}$ & 0.177 & $1.02 \times 10^{-2}$ \\
\hline Melt Pool Width $(\mu \mathrm{m})$ & 125.1 & 1.70 & 142.7 & 1.55 \\
\hline Melt Pool Depth $(\mu \mathrm{m})$ & 36.4 & 0.95 & 37.4 & 0.91 \\
\hline
\end{tabular}

\section{Reduced Order - Simulated Powder}

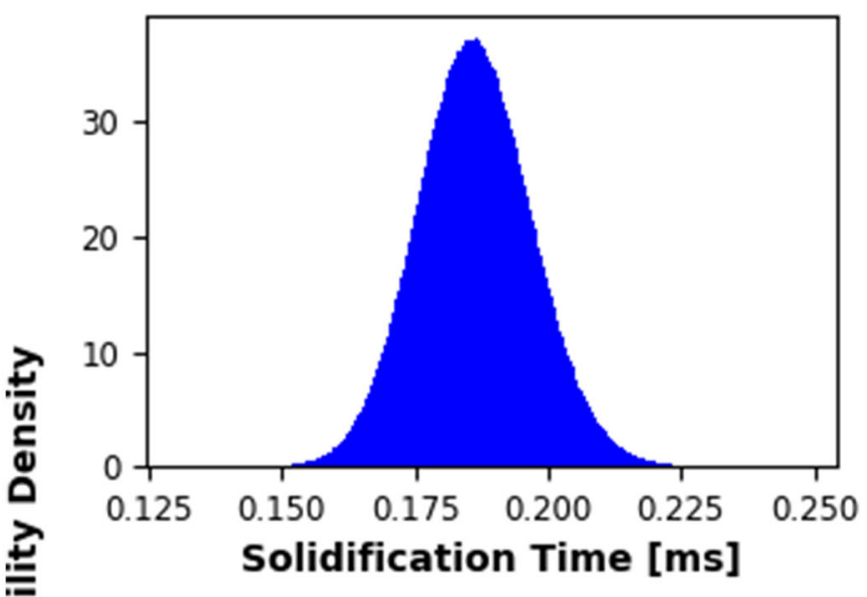

(a)

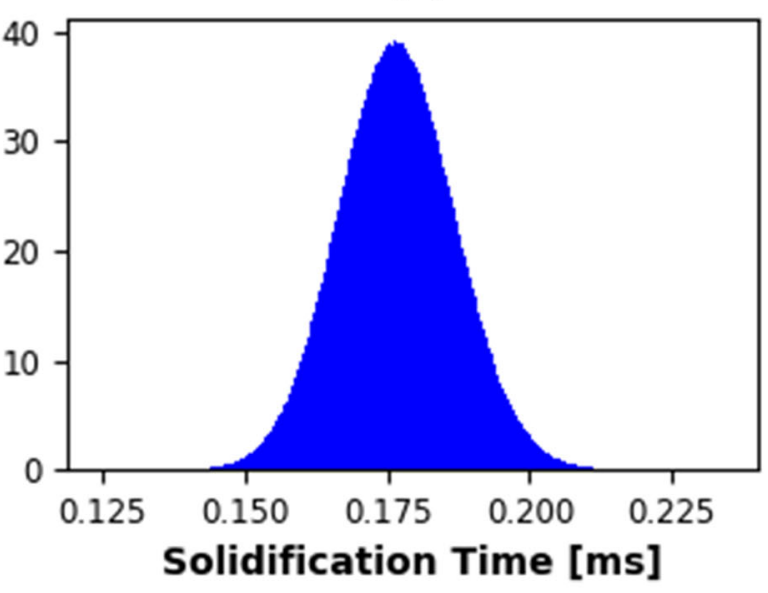

(b)

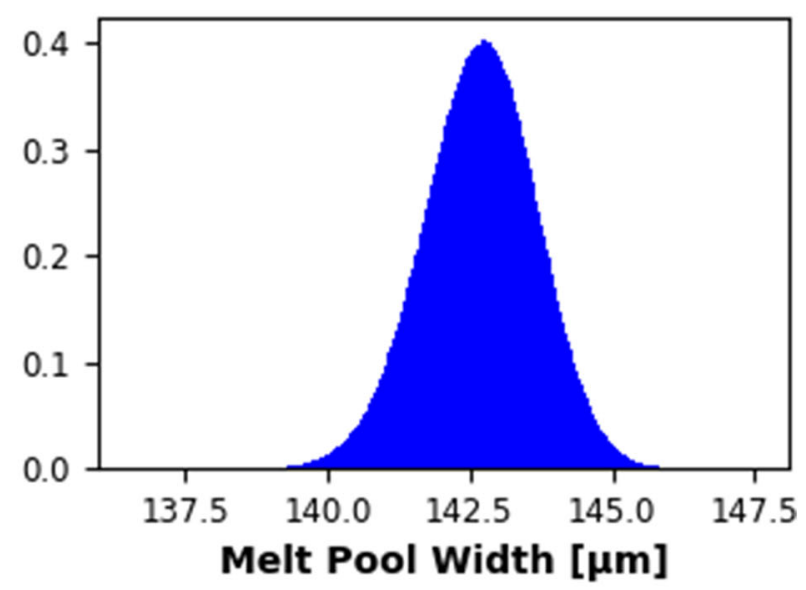

(c)

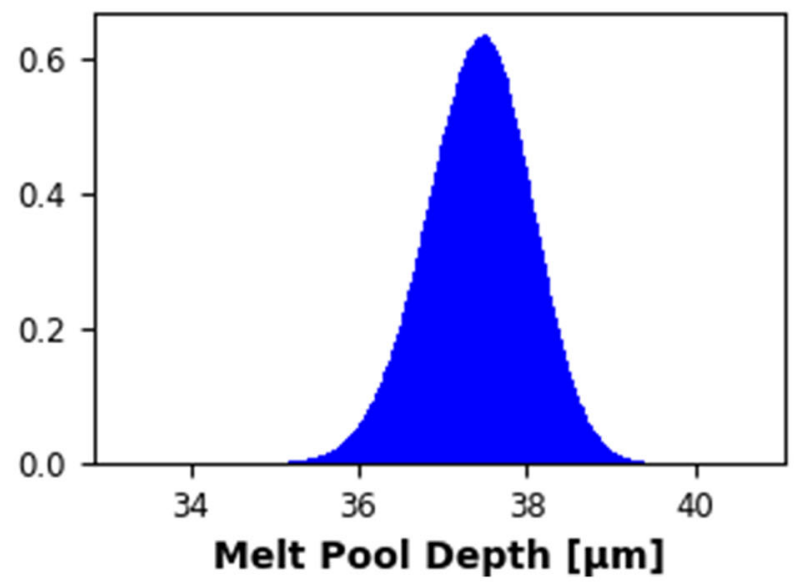

(d)

Fig. 9-Probability distribution functions of the solidification time at the surface $(a)$ and sub-surface $(b)$, and melt pool dimensions $(c, d)$ for the reduced-order analysis when simulating a powder layer. 


\section{Comparison - No Powder}

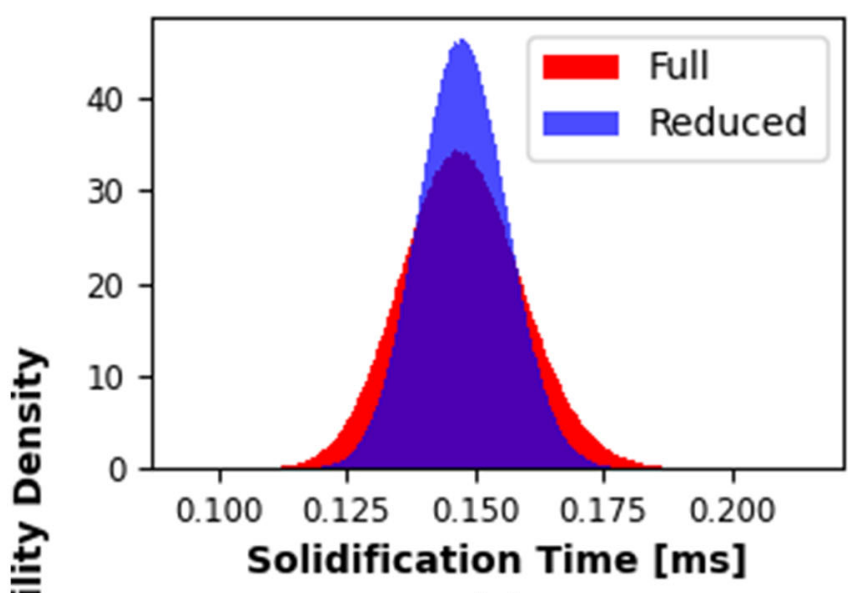

(a)

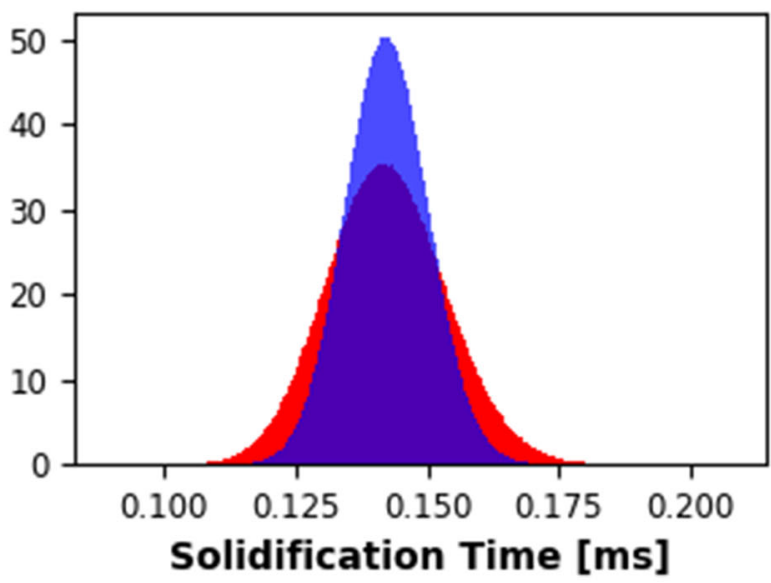

(b)

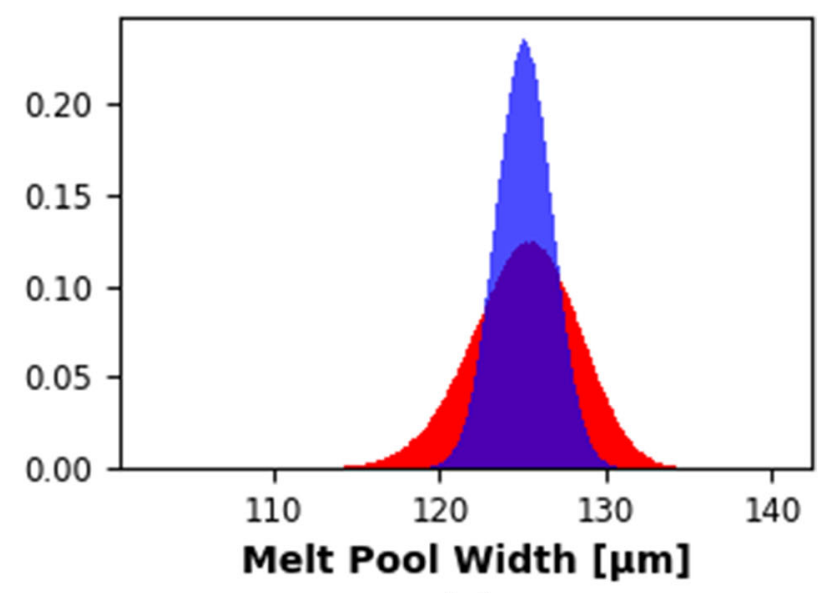

(c)

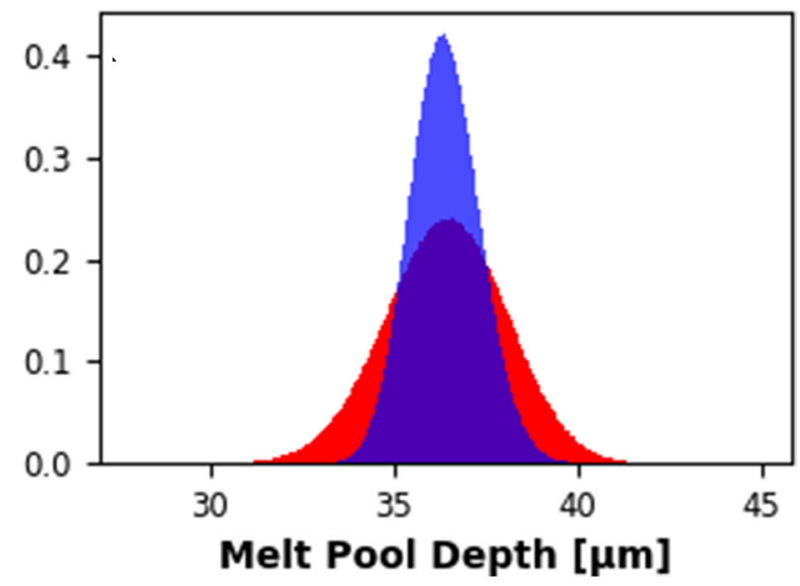

(d)

Fig. 10 - Comparison of the probability distribution functions of the solidification time at the surface $(a)$ and sub-surface $(b)$, and melt pool dimensions $(c, d)$ for the full and reduced-order analysis when not considering the powder layer.

The current study quantifies the propagation of uncertainty in thermophysical properties, process parameters, and model calibration variables, and identifies the most influential parameters on model output uncertainty. While the uncertainty and its effect can be quantified, reducing these effects is difficult. Reduction in uncertainty in thermophysical properties, e.g., specific heat, requires more accurate (and more expensive) measurement techniques. Uncertainty in laser power might be reduced by improvements in the laser pumping source or controllers. Finally, the uncertainty in laser absorption efficiency may be reduced by basing it on a physical model rather than using it as a parameter to calibrate the model to experimental results. Such an approach would, of course, require reliable experimental measurements of the optical properties of the liquid metal surface.

The uncertainty in solidification time may be further propagated by using it as input uncertainty for microstructural prediction models (e.g., cellular automata or phase field) to establish uncertainty bounds on microstructural features such as grain size and crystallographic texture. Finally, the methodology detailed in this work is not restricted to the conduction dominate regime. By using the observations associated with the reduced-order analysis, a useful extension of this work is 


\section{Comparison - Simulated Powder}

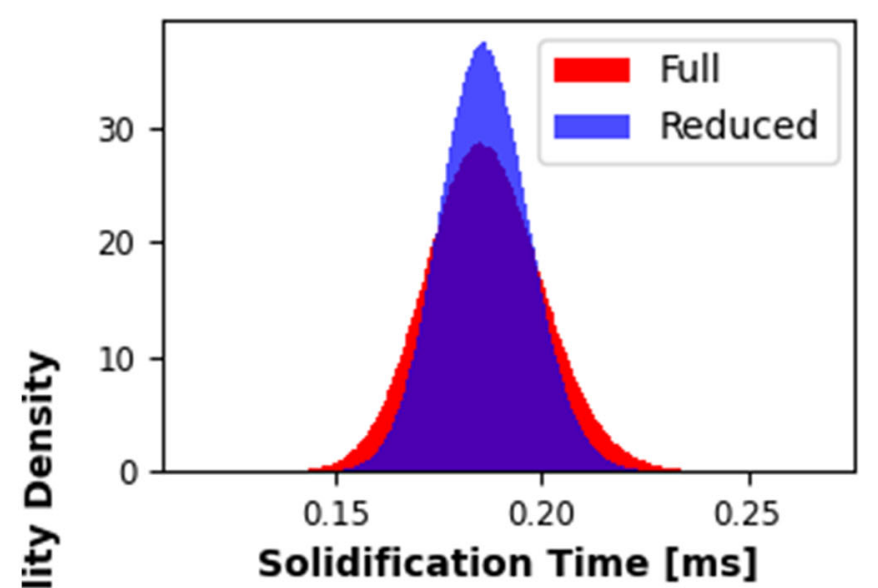

(a)

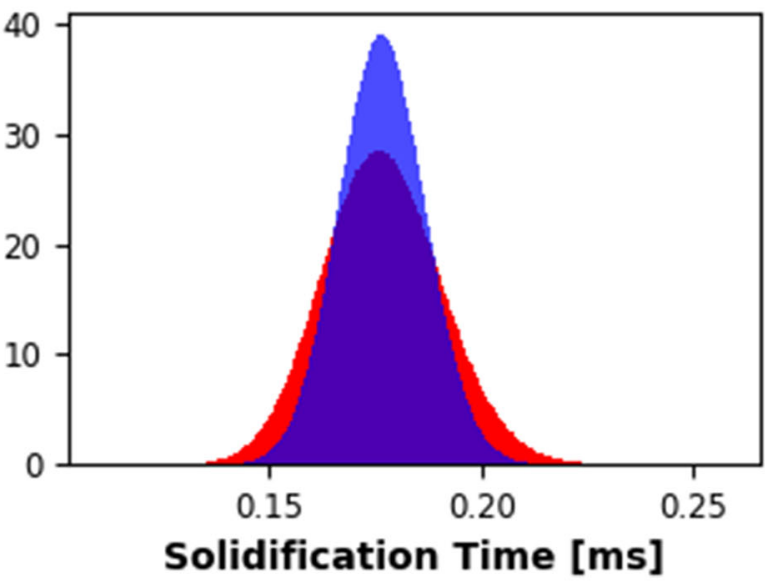

(b)

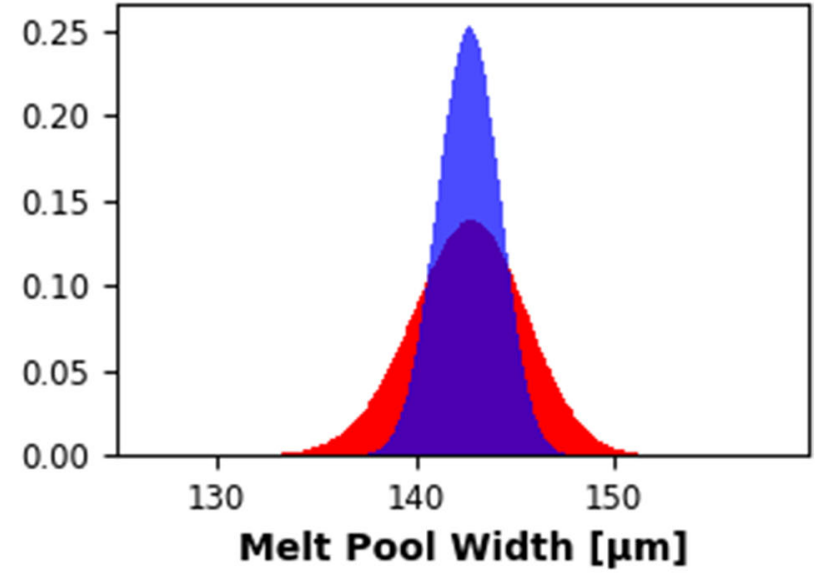

(c)

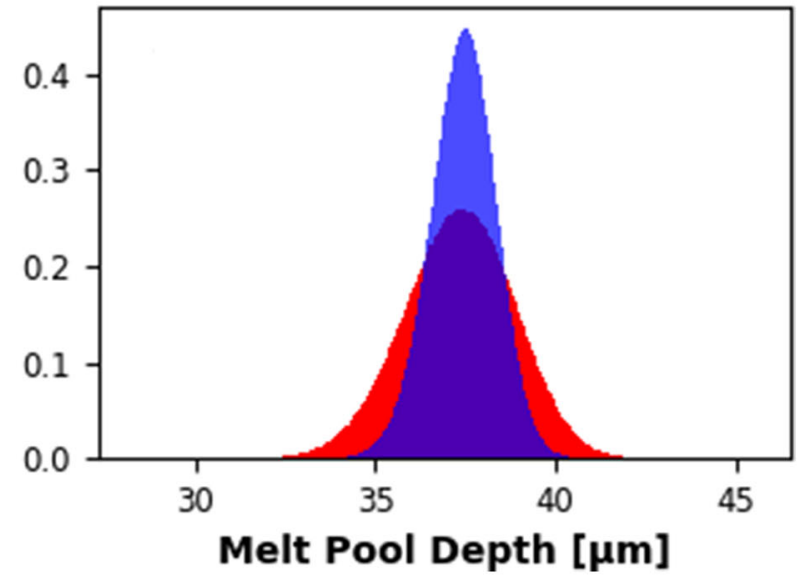

(d)

Fig. 11-Comparison of PDFs for solidification time at the surface $(a)$ and sub-surface $(b)$, and melt pool dimensions $(c, d)$ when simulating a powder layer.

Table VIII. Statistics for the Monte Carlo Sampling of the Comprehensive and Reduced-Order Uncertainty Analysis When the Powder is not Modeled and the Mixed Properties Powder Bed Models

\begin{tabular}{|c|c|c|c|c|c|}
\hline & & \multicolumn{2}{|c|}{ Full } & \multicolumn{2}{|c|}{ Reduced-Order } \\
\hline & & Mean & $2 \sigma$ & Mean & $2 \sigma$ \\
\hline \multirow[t]{4}{*}{ No Powder Model } & $t_{\mathrm{s}}(\mathrm{ms})($ surface $)$ & 0.148 & $1.17 \times 10^{-2}$ & 0.148 & $0.86 \times 10^{-2}$ \\
\hline & $t_{\mathrm{s}}(\mathrm{ms})$ (sub-surface) & 0.142 & $1.13 \times 10^{-2}$ & 0.142 & $0.79 \times 10^{-2}$ \\
\hline & width $(\mu \mathrm{m})$ & 125.1 & 3.23 & 125.09 & 1.70 \\
\hline & depth $(\mu \mathrm{m})$ & 36.4 & 1.65 & 36.41 & 0.95 \\
\hline \multirow[t]{4}{*}{ Simulated Powder Model } & $t_{\mathrm{s}}(\mathrm{ms})($ surface $)$ & 0.186 & $1.47 \times 10^{-2}$ & 0.186 & $1.08 \times 10^{-2}$ \\
\hline & $t_{\mathrm{s}}(\mathrm{ms})$ (sub-surface) & 0.177 & $1.40 \times 10^{-2}$ & 0.177 & $1.02 \times 10^{-2}$ \\
\hline & width $(\mu \mathrm{m})$ & 142.7 & 2.89 & 142.68 & 1.55 \\
\hline & depth $(\mu \mathrm{m})$ & 37.3 & 1.53 & 37.427 & 0.91 \\
\hline
\end{tabular}

to investigate the uncertainty in model inputs under processing conditions (high power and low scan speeds) where fluid flow and convective heat transfer in the molten pool cannot be neglected.

\section{ACKNOWLEDGMENTS}

The authors would like to thank John Coleman for his guidance with regards to the numerical model and Miroslav Stoyanov for his assistance with TASMA- 
NIAN. This work was supported by the Exascale Computing Project (17-SC-20-SC), a collaborative effort of the U.S. DOE Office of Science and the NNSA. This manuscript has been authored by UT-Battelle, LLC under Contract No. DE-AC05-00OR22725 with the U.S. Department of Energy. The United States Government retains and the publisher, by accepting the article for publication, acknowledges that the United States Government retains a non-exclusive, paid-up, irrevocable, world-wide license to publish or reproduce the published form of this manuscript, or allow others to do so, for United States Government purposes. The Department of Energy will provide public access to these results of federally sponsored research in accordance with the DOE Public Access Plan (http://energy.gov/downloads/doe-p ublic-access-plan).

\section{REFERENCES}

1. H. Lee, C.H.J. Lim, M.J. Low, N. Tham, V.M. Murukeshan, and Y.J. Kim: Int. J. Precis. Eng. Manuf. Green Tech., 2017, vol. 4, pp. $307-22$.

2. T. DebRoy, H.L. Wei, J.S. Zuback, T. Mukherjee, J.W. Elmer, J.O. Milewski, A.M. Beese, A. Wilson-Heid, A. De, and W. Zhang: Prog. Mater. Sci., 2018, vol. 92, pp. 112-224.

3. I. Yadroitsev and I. Smurov: Phys. Procedia, 2011, vol. 12A, pp. $264-70$

4. W.E. King, H.D. Barth, V.M. Castillo, G.F. Gallegos, J.W. Gibbs, D.E. Hahn, C. Kamath, and A.M. Rubenchik: J. Mater. Process. Technol., 2014, vol. 214, pp. 2915-25.

5. M. Bayat, A. Thanki, S. Mohanty, A. Witvrouw, S. Yang, J. Thorborg, N.S. Tiedje, and J.H. Hattel: Addit. Manuf., 2019, vol. 30 , art. no. 100835 .

6. A.A. Martin, N.P. Calta, S.A. Khairallah, J. Wang, P.J. Depond, A.Y. Fong, V. Thampy, G.M. Guss, A.M. Kiss, K.H. Stone, C.J. Tassone, J.N. Weker, M.F. Toney, T. van Buuren, and M.J. Matthews: Nat. Commun., 2019, vol. 10 (1), p. 1987.

7. B.M. Morrow, T.J. Lienert, C.M. Knapp, J.O. Sutton, M.J. Brand, R.M. Pacheco, V. Livescu, J.S. Carpenter, and G.T. Gray, III: Metall. Mater. Trans. A, 2018, vol. 49A, pp. 3637-50.

8. M. Iebba, A. Astarita, D. Mistretta, I. Colonna, M. Liberini, F. Scherillo, C. Pirozzi, R. Borrelli, S. Franchitti, and A. Squillace: $J$. Mater. Eng. Perform., 2017, vol. 26, pp. 4138-47.
9. G. Marchese, G. Basile, E. Bassini, A. Aversa, M. Lombardi, D. Ugues, P. Fino, and S. Biamino: Materials, 2018, vol. 11, p. 106.

10. D. Wang, Z. Wang, K. Li, J. Ma, W. Liu, and Z. Shen: Mater. Des., 2019, vol. 162, pp. 384-93.

11. S. Coeck, M. Bisht, J. Plas, and F. Verbist: Addit. Manuf., 2019, vol. 25 , pp. $347-56$.

12. T. Mukherjee, W. Zhang, and T. DebRoy: Comput. Mater. Sci., 2017, vol. 126, pp. 360-72

13. N. Sanaei, A. Fatemi, and N. Phan: Mater. Des., 2019, vol. 182, art. no. 108091

14. L. Johnson, M. Mahmoudi, B. Zhang, R. Seede, X. Huang, J.T. Maier, H.J. Maier, I. Karaman, A. Elwany, and R. Arróyave: Acta. Mater., 2019, vol. 176, pp. 199-210.

15. M. Brandt: Laser Additive Manufacturing: Materials, Design, Technologies, and Applications, Woodhead Publishing, Cambridge, UK, 2016.

16. I. Yadroitsev: Selective Laser Melting: Direct Manufacturing of 3D-Objects by Selective Laser Melting of Metal Powders, LAP Lambert Academic Publishing, Saarbrücken, 2009.

17. J. Coleman, A. Plotkowski, B. Stump, N. Raghavan, A. Sabau, M.J.M. Krane, J. Heigel, R. Ricker, L. Levine, and S.S. Babu: $J$. Heat Transf., 2020, vol. 142 (12), art. no. 122201.

18. M. Binkley, Microstructure Development in Multi-pass Laser Melting of AISI 8620 Steel, M.S. thesis, School of Materials Engineering, Purdue University, 2020.

19. Z. Morrow and M. Stoyanov: J. Sci. Comput., 2019, vol. 42 (4), pp. $2436-60$.

20. M. Stoyanov: User Manual: Tasmanian Sparse Grids, Tech. Report ORNL/TM-2015/596, Oak Ridge National Laboratory, Oak Ridge, TN, 2015.

21. M. Stoyanov: Sparse Grids and Applications-Miami 2016, Springer, New York, 2018, pp. 175-99.

22. M. Stoyanov, D. Lebrun-Grandie, J. Burkardt, and D. Munster, 2013, https://github.com/ORNL/Tasmanian. Accessed 3 January 2021.

23. M. Stoyanov and C.G. Webster: Comput. Math. Appl., 2016, vol. 71 (11), pp. 2449-65.

24. F. Rösler and D. Brüggemann: Heat Mass Transf., 2011, vol. 47, pp. 1027-33.

25. A.V. Gusarov and E.P. Kovalev: Phys. Rev. B, 2009, vol. 80, pp. 024202-1-024202-15.

26. A. Plotkowski and M. J. M. Krane: LMPC 2017, Proc. Int. Symp. Liq. Met. Process. Cast., 2017.

27. K. Fezi and M.J.M. Krane: Metall. Mater. Trans. B, 2018, vol. 49, pp. 4759-70.

28. D.C. Deisenroth, S. Mekhontsev, and B. Lane: Proc. SPIE 11271, Laser 3D Manufacturing VII, 112710L, 2020.

Publisher's Note Springer Nature remains neutral with regard to jurisdictional claims in published maps and institutional affiliations. 